\title{
Dynamic Replication of Non-Maturing Assets and Liabilities
}

\author{
Michael Schürle \\ Institute for Operations Research and Computational Finance, \\ University of St. Gallen, Bodanstr. 6, CH-9000 St. Gallen, Switzerland \\ michael.schuerle@unisg.ch
}

\section{Introduction}

Non-maturing assets and liabilities (NoMALs) are positions in a bank's balance with no contractual maturity such as savings or sight deposits. Clients have the option to add or withdraw investments or credits while the bank may adjust the customer rate anytime. It is often observed that the volume of NoMALs fluctuates significantly as clients react to changes in the customer rate or to the relative attractiveness of alternative investment opportunities.

Although there is no explicit maturity, banks must assign a fix maturity profile to a NoMAL: (a) It defines the transfer price at which the margin is split between a retail unit and the treasury. (b) The treasury manages the interest rate risk based on such a transformation of uncertain cash flows into (apparently) certain ones. To this end, most banks construct a replicating portfolio of fixed-income instruments where maturing tranches are always renewed at constant weights. The latter are determined from historical data by minimizing the tracking error between the cash flows of the portfolio (coupon payments) and those of the NoMAL (given by client rate and volume changes) during the sample period. Then, the transfer price is equivalent to the average margin between the yield on the portfolio and the client rate.

In the practical implementation, one can often observe that the portfolio composition and the corresponding margin are sensitive to the sample period. This induces the considerable model risk of an improper transformation of the variable position with direct implications on the "correct" transfer price and hedge-ability of the position. One possible result may also be that the replicating portfolio with the least volatile margin provides an income that does not cover the costs of holding the account. Then, it cannot be seen as the strategy with the lowest risk if it leads to a sure loss.

As an alternative to fitting the portfolio composition to a single historic scenario, this paper proposes a multistage stochastic programming model where the optimal allocation of new instruments is derived from some thousand scenarios of future interest rates, client rates and volumes. Instead of 
constant portfolio weights, the amounts invested or financed in each maturity are frequently adjusted taking future transactions and their impact on today's decision explicitly into account. Furthermore, risk is defined as the downside deviation of not meeting a specified minimum target for the margin.

\section{Formulation as Multistage Stochastic Program}

\subsection{Notation}

For simplicity, the following description is restricted to a model for deposits. An equivalent formulation for active products can easily be derived when investing is replaced by borrowing and vice versa. Let $D$ be the longest maturity used for the construction of the replicating portfolio. $\mathcal{D}=\{1, \ldots, D\}$ denotes the set of dates where fixed-income securities held in the portfolio mature. The maturities of traded standard instruments that can be used for investment are given by the set $\mathcal{D}^{+} \subseteq \mathcal{D}$. Furthermore, instruments in the set $\mathcal{D}^{-} \subseteq \mathcal{D}$ may be squared prior to maturity (modelled as borrowing funds of the corresponding maturities).

It is assumed that the joint evolution of random data (market rates, client rate and volume of the NoMAL) is driven by a stochastic process $\boldsymbol{\omega}:=\left(\boldsymbol{\omega}_{t} ; t=1, \ldots, T\right)$ in discrete time defined on a probability space $(\Omega, \mathcal{F}, P)$ which satisfies the usual assumptions (with $\left.\Omega:=\Omega_{1} \times \ldots \times \Omega_{T}\right)$. The random vector $\boldsymbol{\omega}_{t}:=\left(\boldsymbol{\eta}_{t}, \boldsymbol{\xi}_{t}\right) \in \Omega_{t}^{\eta} \times \Omega_{t}^{\xi}=: \Omega_{t} \subseteq \mathbb{R}^{K+L}$ can be decomposed into two components: $\boldsymbol{\eta}_{t} \in \Omega_{t}^{\eta} \subseteq \mathbb{R}^{K}$ is equivalent to the state variables of a $K$-factor term structure model and controls market rates, client rate and volume. The latter is also affected by the process $\boldsymbol{\xi}_{t} \in \Omega_{t}^{\xi} \subseteq \mathbb{R}^{L}$ that may represent additional economic factors with impact on the savings volume or a residual variable for non-systematic variations. The relevant stochastic coefficients in the optimization model at stage $t$ depend on the histories of observations $\boldsymbol{\eta}^{t}:=\left(\boldsymbol{\eta}_{1}, \ldots, \boldsymbol{\eta}_{t}\right)$ and $\boldsymbol{\omega}^{t}:=\left(\boldsymbol{\omega}_{1}, \ldots, \boldsymbol{\omega}_{t}\right)$ :

$r_{t}^{d,+}\left(\boldsymbol{\eta}^{t}\right)$ bid rate per period for investing in maturity $d \in \mathcal{D}^{+}$,

$r_{t}^{d,-}\left(\boldsymbol{\eta}^{t}\right)$ ask rate per period for financing in maturity $d \in \mathcal{D}^{-}$,

$c_{t}\left(\boldsymbol{\eta}^{t}\right) \quad$ client rate paid per period for holding the deposit account,

$v_{t}\left(\boldsymbol{\omega}^{t}\right) \quad$ volume of the non-maturing account position.

In the sequel, the dependency of the coefficients on $\boldsymbol{\omega}^{t}$ or $\boldsymbol{\eta}^{t}$ will not be stressed in the notation for simplicity. Interest rates, client rate and volume for $t=0$ are deterministic and can be obtained from current market observations.

\subsection{Optimization Model}

At each stage $t=0, \ldots, T$, where $T$ denotes the planning horizon, decisions are made on the amount $x_{t}^{d,+}$ invested in maturity $d \in \mathcal{D}^{+}$and the amount $x_{t}^{d,-}$ financed in maturity $d \in \mathcal{D}^{-}$. The totally invested volume of the replicating 
portfolio, i.e., the sum of investments minus borrowings over all stages up to $t$ plus instruments held in the initial portfolio that have not yet matured, has to match the stochastic volume of the position at all points in time:

$$
\sum_{\tau=0}^{t} \sum_{\substack{d \in \mathcal{D}^{+} \\ d>\tau}} x_{t-\tau}^{d,+}-\sum_{\tau=0}^{t} \sum_{\substack{d \in \mathcal{D}^{-} \\ d>\tau}} x_{t-\tau}^{d,-}+\sum_{d=t+2}^{D} x_{-1}^{d}=v_{t}
$$

where $x_{-1}^{d}$ denotes a position in the initial portfolio maturing at time $d$. Negative holdings are not allowed, i.e., the amount squared in a certain maturity must not exceed the investments made earlier with the same maturity date:

$$
x_{t}^{d,-} \leq \sum_{\substack{\tau=1 \\ d+\tau \in \mathcal{D}^{+}}}^{t} x_{t-\tau}^{d+\tau,+}-\sum_{\substack{\tau=1 \\ d+\tau \in \mathcal{D}^{-}}}^{t} x_{t-\tau}^{d+\tau,-}+x_{-1}^{t+1+d} \quad \forall d \in \mathcal{D}^{-} .
$$

Transactions in $t$ result in a surplus $x_{t}^{S}$ that is defined as the difference between the income from the positions held in the replicating portfolio which have not matured minus the costs of holding the account:

$$
x_{t}^{S}=\sum_{\tau=0}^{\tau^{+}} \sum_{\substack{d \in \mathcal{D}^{+} \\ d>\tau}} r_{t-\tau}^{d,+} \cdot x_{t-\tau}^{d,+}-\sum_{\tau=0}^{\tau^{-}} \sum_{\substack{d \in \mathcal{D}^{-} \\ d>\tau}} r_{t-\tau}^{d,-} \cdot x_{t-\tau}^{d,-}+c f_{-1}^{t+2}-\left(c_{t}+\alpha_{0}\right) \cdot v_{t},
$$

where $\tau^{+}:=\min \left\{t, \max \left\{\mathcal{D}^{+}\right\}-1\right\}, \tau^{-}:=\min \left\{t, \max \left\{\mathcal{D}^{-}\right\}-1\right\}$ and $c f_{-1}^{t}$ is the sum of all coupon payments from positions in the initial portfolio with maturity $t$. The costs of holding the position consist not only of payments made to clients but also of non-interest expenses of serving the deposit. Hence, the forth term on the right-hand-side of (3) contains the constant $\alpha_{0}$ to specify a target for the margin that must be achieved in addition to the client rate. The objective of the stochastic program is to minimize the expected downside deviation of not meeting the specified target over all stages:

$$
\begin{array}{lll}
\min & \int_{\Omega} \sum_{t=0}^{T} x_{t}^{M} d P(\boldsymbol{\omega}) \\
\text { s.t. } & \text { equations }(1)-(3) \\
& x_{t}^{M} \geq-x_{t}^{S} \\
& 0 \leq x_{t}^{d,+} \leq \ell^{d,+} \quad \mathcal{F}_{t} \text {-measurable } \quad \forall d \in \mathcal{D}^{+} \\
& 0 \leq x_{t}^{d,-} \leq \ell^{d,-} \quad \mathcal{F}_{t} \text {-measurable } \quad \forall d \in \mathcal{D}^{-} \\
& x_{t}^{S} \in \mathbb{R} ; x_{t}^{M} \geq 0 \quad \mathcal{F}_{t} \text {-measurable }
\end{array}
$$

Decision and state variables herein for $t>0$ are stochastic since they depend on observations of the random data process $\boldsymbol{\omega}^{t}$ up to time $t$. Therefore, they are adapted to the filtration $\mathcal{F}_{t}$ that specifies the information structure, i.e., 
they are taken only with respect to the information available at this time (nonanticipativity). All constraints must hold almost surely (a.s.), i.e., for all $\boldsymbol{\omega} \in \Omega$ except for sets with zero probability. A common way to make the stochastic program (4) computationally tractable is the generation of a scenario tree as an approximation of the vector stochastic process $\boldsymbol{\omega}$ in $(\Omega, \mathcal{F}, P)$. The resulting deterministic problem is a large-scale linear program that can be solved with standard algorithms like Cplex (see [2] for a recent introduction to stochastic programming methods).

\section{Scenario Generation}

The model for the stochastic evolution of risk factors consists of three components: Its core is a term structure model with $K=2$ factors for the level and steepness of the yield curve that fluctuate around long term means $\theta_{1}:=\theta$ and $\theta_{2}:=0$. Their dynamics are described by the stochastic differential equations

$$
d \eta_{i t}=\kappa_{i}\left(\theta_{i}-\eta_{i t}\right) d t+\sigma_{i} d z_{i t}, \quad i=1,2,
$$

where $d z_{1}$ and $d z_{2}$ are the increments of two uncorrelated Wiener processes. $\kappa_{i}$ controls the speed at which the $i$-th factor reverts to its mean and $\sigma_{i}$ controls the instantaneous volatility. It is known that under specification (5) both factors are normally distributed. This is an extension of the well-known Vasicek model [5]. Explicit formulae exist to derive the yield curve as a function of the two factors. Compared to alternative term structure models in an investigation for the Swiss market [1], this turned out to be the most suitable one for scenario generation. Parameter estimates are derived from historic interest rates using the maximum likelihood method described in [3].

The second component models the client rate as a (deterministic) function of the level factor $\eta_{1}$ to reflect specific characteristics of the relevant NoMAL and the dependency on interest rates. In case of Swiss savings accounts, banks adjust the customer rate only at discrete increments, typically a multiple of 25 basis points (bp). Furthermore, the adjustment is asymmetric when market rates rise or fall, and there is a (political) cap where higher rates are no longer passed to depositors. Let $\delta_{0}<\ldots<\delta_{n}$ be the possible increments (including the value 0$)$ and $\gamma_{0}<\gamma_{1}<\ldots<\gamma_{n}<\gamma_{n+1}$ some threshold values $\left(\gamma_{0}:=-\infty\right.$, $\left.\gamma_{n+1}:=\infty\right)$. Then, the client rate changes by $\Delta c_{t}=\delta_{i}$ if the latent control variable $c_{t}^{*}=\beta_{0} c_{t-1}+\beta_{1} \eta_{1, t}+\ldots+\beta_{m+1} \eta_{1, t-m}$ is realized between the threshold values $\gamma_{i}$ and $\gamma_{i+1}$. The coefficients of the control variable process and the threshold values are estimated jointly from historical data using a maximum likelihood procedure.

Finally, relative changes in the volume $v_{t}$ are modelled by

$$
\ln v_{t}=\ln v_{t-1}+e_{0}+e_{1} t+e_{2} \eta_{1 t}+e_{3} \eta_{2 t}+\xi_{t} .
$$

The constant $e_{0}$ and the time component $e_{1} t$ reflect that the volume exhibits a positive trend (nominal balances can be expected to increase in the long 
run due to inflation). The factors $\eta_{1}$ and $\eta_{2}$ of the term structure model are included as explanatory variables because market rates influence the account when clients transfer volume from or to other interest rate sensitive investments like savings certificates. An additional stochastic factor $\xi(L=1)$ which is uncorrelated with the market rate model factors takes into account that the latter do not fully explain the observed evolution of the balance. Equation (6) can easily be estimated by ordinary least squares regression, and the volatility $\sigma_{\xi}$ of the residuum factor $\xi$ is immediately derived from the standard error.

For the generation of a scenario tree, the multivariate normal distribution of the random vector $\boldsymbol{\omega}_{t}:=\left(\eta_{1 t}, \eta_{2 t}, \xi_{t}\right)$ at $t=1, \ldots, T$ is approximated by a multinomial distribution. This provides finite sets of samples and corresponding probabilities at each stage that preserve the expectations and covariance matrix implied by the term structure and volume model (5)-(6) after a transformation (details are described in [4]).

\section{Results}

The model was tested with client rate and volume data of a real Swiss savings deposit position for the period January 1989 to December 2001. Market rates during this time showed a phase of inverse term structures at high level at the beginning, followed by an abrupt change to a period of low interest rates and normal yield curves in the second half. Interbank market instruments with maturities from 1 to 10 years were used for the construction of the replicating portfolio. Positions were squared only if the amount of maturing tranches was not sufficient to compensate a drop in volume. The margin target for the downside minimization was set to $\alpha_{0}=200 \mathrm{bp}$. Figure 1 shows that the stochastic programming model was able to meet this value almost anytime during the sample period while the margin of a static replicating portfolio that was calculated as benchmark collapsed after the drop in market rates.

\begin{tabular}{lccc}
\hline model & avg. margin & std. dev. & avg. maturity \\
\hline dynamic replication & $2.23 \%$ & $0.32 \%$ & 2.37 yrs. \\
static benchmark & $1.93 \%$ & $0.49 \%$ & 1.81 yrs. \\
\hline
\end{tabular}

According to the table, the average margin was increased by $30 \mathrm{bp}$ while simultaneously the volatility was reduced. Therefore, the dynamic strategy provides the more efficient replication. The corresponding portfolio has also a higher duration, indicating that the "true" maturity of the NoMAL position is approx. 0.5 years longer than implied by the static replication. Note that an extension of the duration by half a year in a portfolio with constant weights would yield at most a gain of $10 \mathrm{bp}$ at larger volatility. This allows the conclusion that the higher margin achieved here can mainly be attributed to the added value of dynamic management. 


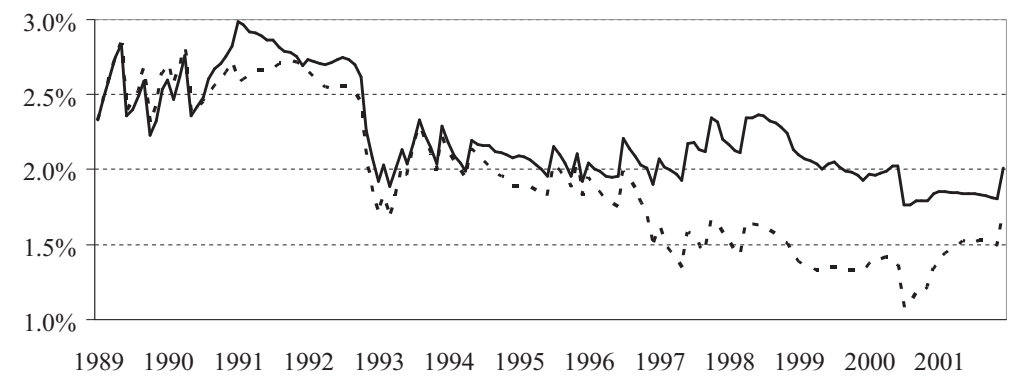

Fig. 1. Margin evolution: Dynamic (solid) vs. static replication (dashed)

\section{Conclusions and Outlook}

Dynamic strategies have turned out to be more efficient for the replication of variable banking products than the classical static approach. A further analysis reveals that the portfolio compositions are by far less sensitive to changes in the input parameters due to different sample periods. This observation is typical for stochastic programming models [3]. From a practical point of view, no other data are required for the calibration of the risk factor models than for the determination of the (constant) weights in static replication.

The description of the stochastic programming model here was restricted to a basic version (4). The implementation of the complete model contains also alternative objective functions (e.g., minimization of volatility instead of downside risk) and additional constraints for the portfolio structure, admissible transactions and risk. Current research is directed towards the assessment of "end effects", i.e., the truncation of the planning horizon at some finite number of stages $T$ although it is infinite for NoMAL management by definition. Approximations of the true infinite horizon problem are being developed that allow a quantification of the impact of events after time $T$ on earlier decisions. First results imply that these techniques may have some potential for an additional improvement of the model performance and robustness.

\section{References}

1. Frauendorfer K, Schürle M (2000) Term structure models in multistage stochastic programming. Annals of Operations Research 100:189-209

2. Kall P, Mayer J (2005) Stochastic Linear Programming. Springer, New York

3. Schürle M (1998) Zinsmodelle in der stochastischen Optimierung. Haupt, Berne

4. Siede H (2000) Multi-Period Portfolio Optimization. PhD thesis, University of St. Gallen

5. Vasicek O (1977) An equilibrium characterization of the term structure. Journal of Financial Economics 5:177-188 